\title{
FCC: Building on the shoulders of giants
}

\author{
Stephen Myers ${ }^{\mathrm{a}}(\mathbb{D}$ \\ CERN/ADAM SA, Geneva, Switzerland
}

Received: 31 August 2021 / Accepted: 8 October 2021

(C) The Author(s) 2021, corrected publication 2022

\begin{abstract}
The plethora of open questions in particle physics, the new chapter opened by the Higgs boson, and the lack of clear theoretical guidance as to where new theory could lie call for a broad and diverse experimental programme boosting the intensity and energy-frontier. The proposed FCC-integrated programme consisting of a luminosity-frontier highest-energy lepton collider followed by an energy-frontier hadron collider promises the most far-reaching particle physics programme that foreseeable technology can deliver. In this essay, particular emphasis is given to the lessons from the predecessor of the LHC, LEP, which was commissioned in 1989 and finished operation in November 2000.
\end{abstract}

\section{Introduction}

The recent update of the European Strategy for particle physics sets a concrete vision for the future of particle physics and the tools that should be developed to continue exploring nature at the subatomic scale. The strategy acknowledges a number of outstanding questions that need to be addressed in the future programme of the LHC and in the planning of a post-LHC research infrastructure like the proposed future circular collider (FCC). Questions related to the composition of dark matter and the existence of a dark sector in our Universe, the origin of the observed asymmetry between matter and antimatter and the neutrino masses call for more data and guide the design of new experiments. Moreover, the study of the recently discovered Higgs boson-with its notoriously hard to explain small mass - that could potentially be linked to these questions is identified as the key priority for a post-LHC collider.

The plethora of open questions in particle physics, the new chapter opened by the Higgs boson and the lack of clear theoretical guidance as to where new theory could lie call for a broad and diverse experimental programme boosting the intensity and energy-frontier. The proposed FCC-integrated programme consisting of a luminosity-frontier highest-energy lepton collider followed by an energy-frontier hadron collider promises the most far-reaching particle physics programme that foreseeable technology can deliver. In this essay, particular emphasis is given to the lessons from the predecessor of the LHC, LEP, which was commissioned in 1989 and finished operation in November 2000.

The FCC-ee design profits from experience gained in many previous electron-positron colliders and provides very high-integrated luminosities at collision energies between 88

\footnotetext{
a e-mail: Stephen.Myers@ avo-adam.com (corresponding author)
} 
and $365 \mathrm{GeV}$. The FCC-ee design incorporates many new concepts and schemes tested and proposed in recent lepton colliders:

- a double-ring configuration, obviating the need for complicated separation schemes such as those used in CERN's Large electron-positron collider (LEP), and allowing a large number of bunches per beam, and a concomitant very high luminosity;

- a crab-waist crossing allowing very small values of the vertical $\beta$ function at the collision points;

- a top-up injection scheme permitting the average luminosity to approach the peak luminosity and greatly improving the integrated luminosity.

The concept of the electron synchrotron collider is well-proven and tested. It dates back more than fifty years and has been confirmed in recent high-energy, high-luminosity colliders such as LEP, LEP2, DAФNE, PETRA, PEP-II and KEKB. Circular colliders also allow the possibility of multiple experimental regions which is another great advantage of this particular scheme. In addition, the stabilising effect of strong synchrotron radiation renders circulating beams in high-energy electron synchrotrons almost impervious to many types of perturbations.

In this essay, I will look back at previous colliders and try to highlight the positive as well as the negative lessons learned in terms of their design, construction, installation, commissioning and operation.

These lessons will not only be technical but will include the following aspects:

- choice of location,

- new/modern tunnelling techniques and reuse of excavation material,

- attractive infrastructure location offering a healthy, happy and attractive environment for families and individuals.

- control and availability of resources (human \& financial).

- professional 'commercial' project management including: budget control, reliable schedule/planning, quality assurance, resource savings/optimisation through large-scale/series manufacturing of the accelerator components.

- building an international collaboration with stakeholders from academia and industry.

- the wider socio-economic benefits of a new research infrastructure and the benefits that stem for society including human-capital formation, the training of the next generation of experts in scientific research as well as the industrial and cultural value of such big science projects.

I will also point out and comment on the relative technological 'leaps' made by each new project in terms of beam energy and machine circumference over the past 50 years or so.

Based on the lessons learned, I will make suggestions in a 'Roadmap for Success' that could help to avoid the pitfalls of previous projects.

\subsection{Competition}

As a result of the renewed world-wide interest for $\mathrm{e}^{+} \mathrm{e}^{-}$physics and the pertaining discovery potential since the observation of the Higgs boson at the LHC, the FCC study has competition. Today, there are four $\mathrm{e}^{+} \mathrm{e}^{-}$collider proposals which are designed to study the properties of the Higgs boson and other standard model particles:

- the International Linear Collider (ILC) project with a centre-of-mass energy of $250 \mathrm{GeV}$;

- the Compact Linear Collider (CLIC), where the first-stage centre-of-mass energy point was reduced from 500 to $380 \mathrm{GeV}$; 
Table 1 Sub-set of

electron-positron colliders with which the author had experience

\begin{tabular}{lllll}
\hline $\begin{array}{l}\text { Collider } \\
\text { name }\end{array}$ & $\begin{array}{l}\text { First } \\
\text { operation }\end{array}$ & $\begin{array}{l}\text { Beam } \\
\text { energy } \\
(\mathrm{GeV})\end{array}$ & $\begin{array}{l}\text { Circumference } \\
(\mathrm{km})\end{array}$ \\
\hline SPEAR & 1972 & 4.2 & 0.234 & Lepton \\
DORIS & 1973 & 5.6 & 0.289 & Lepton \\
PETRA & 1978 & 23.4 & 2.3 & Lepton \\
PEP & 1980 & 15 & 2.2 & Lepton \\
TRISTAN & 1987 & 32 & 3.02 & Lepton \\
LEP & 1989 & 104.5 & 26.7 & Lepton \\
SLC & 1989 & 50 & NA & Lepton \\
HERA 1 & 1992 & 30 & 6.3 & Lepton \\
FCC-ee & NA & 182.5 & 98 & Lepton \\
\hline
\end{tabular}

- the Circular Electron Positron Collider (CEPC), in a $100 \mathrm{~km}$ tunnel in China, with centreof-mass energies from 90 to $250 \mathrm{GeV}$;

- and the Future $\mathrm{e}^{+} \mathrm{e}^{-}$Circular Collider in a new $\sim 100 \mathrm{~km}$ tunnel at CERN (FCC-ee, formerly called TLEP) that could span energies from $90 \mathrm{GeV}$ at the $\mathrm{Z}$ pole to above the top threshold $(365 \mathrm{GeV})$.

The FCC-ee delivers the highest luminosity in a clean, well-understood, and predictable environment, at the $\mathrm{Z}$ pole $(91 \mathrm{GeV})$, at the WW threshold $(161 \mathrm{GeV})$, as a Higgs factory $(240 \mathrm{GeV})$, and around the $\mathrm{t} \bar{t}$ threshold (340-365 GeV), to two interaction points while also opening the option for multiple collision points. In addition, due to the possibility of transverse polarisation up to $80 \mathrm{GeV}$ beam energy, it can also provide high precision centreof-mass energy calibration at the $\mathrm{Z}$ and $\mathrm{W}$ energies, a unique feature of circular colliders. The two linear colliders can only offer a single effective interaction point, while the CEPC and ILC propose a green field site; in my opinion, these are distinct disadvantages with respect to the FCC-ee proposal that builds on and profits from the current CERN accelerator site.

\section{Technology and construction leaps}

Over the past 70 years, the energy and circumference of $\mathrm{e}^{+} \mathrm{e}^{-}$colliders has increased with each new project. Table 1 shows the progression of this increase. An interesting observation is that the progression from the SPEAR-DORIS projects in the early $70 \mathrm{~s}$ to PEP-PETRA resulted in factors of $\sim 10$ in circumference and in $\sim 5$ in beam energy. The progression from PEP-PETRA to LEP produced similar values of $\sim 10$ and $\sim 5$ in circumference and beam energy, respectively. However, the maximum beam energy achieved by LEP was in LEP2 which used superconducting cavities. Without the development of this new technology, the LEP energy would have been limited to around $70 \mathrm{GeV}$.

If we now compare the leap from LEP2 to FCC-ee, the values for circumference and energy are $\sim 4$ and $\sim 2$ and are lower but comparable to previous designs due to the use of superconducting cavities. This approximate comparison indicates that the technological leap from LEP2 to FCC-ee is reasonable and should be achievable in a sustainable way. 


\section{Lessons learned from previous colliders}

It may now be instructive to delve into some of the lessons learned from previous successful and unsuccessful collider proposals and projects. I will refer to unsuccessful proposals as they allow us to extract useful lessons learned during the design, approval and construction phases and help us identify the practices/steps that should be avoided in the preparation of a post-LHC collider. Furthermore, by looking at some of the successful projects, we will discuss the lessons that one can carry forward from the commissioning and operation of these projects. These lessons come from personal experience, through my work and intimate experience with the ISR, LEP, LEP2, and the LHC. I have also been involved in advisory boards, reviews, etc., on the following projects: SPEAR, DORIS, PETRA, PEP, SP $\bar{P} S$, Tristan, Tevatron, SLC, SSC, HERA, DA $\Phi$ NE, and RHIC. Few of these past projects present similar challenges to the FCC-ee project, so I will concentrate on those projects which I believe are more relevant and instructive, i.e. LEP and the SLC; SSC and the LHC.

\subsection{LEP and the SLC}

The SLC was a highly innovative project which was in direct competition with the more traditional design of the LEP collider. Both colliders started operation in 1989 with the SLC the first to have collisions. However, since the SLC was the first ever linear collider, the luminosity learning curve was shallow and tortuous, whereas LEP rapidly reached its design luminosity using the well-tried and tested synchrotron collider design and the vast amount of experience gained in previous similar colliders. Consequently, LEP very quickly outperformed the SLC in the production of high luminosity.

In my opinion, the lesson from this story is that the technology leap was too big for a new type of collider which needed to produce data for physics on the same timescale as a more traditional albeit much larger collider that was LEP.

This established LEP/LEP2 as the reference for any future $\mathrm{e}^{+} \mathrm{e}^{-}$ring collider design. In the following, I will go through a list of lessons learned from the experience in the design, construction and 12 years of LEP operation.

\subsection{LEP: the positive lessons}

The positive lessons learned from operation of LEP are numerous and are listed below:

- Ultra-precise beam energy determination (through a combination of software tools, new techniques and skilled and experienced operators)

- Collisions with record beam-beam tune shifts. The influence of the transverse damping time on the achievable beam-beam tune shift and the concomitant peak luminosity.

- Experience gained in running a very large collider, with continuous improvements in operational procedures for high efficiency, e.g. orbit optimisation in long machines, alignment, ground motion and emittance stability in deep tunnels.

- Design and efficient operation of a large SC RF system with 288 cavities and a large cryogenic system.

- Highly flexible beam optics with multiple changes in the betatron phase advance per cell ranging from $60^{\circ} / 60^{\circ}$ to $102^{\circ} / 90^{\circ}$ and $102^{\circ} / 45^{\circ}$.

- Real-time feedback on beam parameters (orbit, tune, instabilities etc.) which has been proven essential for the safe and efficient operation of very large colliders.

- A test bed for the elite training of a new generation of leaders in accelerator and detector physics and engineering. 
Table 2 LEP performance parameters

\begin{tabular}{llll}
\hline Parameter & Design $(55 / 95 \mathrm{GeV})$ & Achieved $(46 / 98 \mathrm{GeV})$ & Comment \\
\hline Bunch current & $0.75 \mathrm{~mA}$ & $1.0 \mathrm{~mA}$ & \\
Total beam current & $6.0 \mathrm{~mA}$ & $8.4 / 6.2 \mathrm{~mA}$ & \\
Vertical beam-beam parameter & 0.03 & $0.045 / 0.083$ & \\
Emittance ratio & $4.0 \%$ & $0.4 \%$ & $\times 10$ \\
Maximum luminosity & $16 / 27 \times 10^{30} \mathrm{~cm}^{-2} \mathrm{~s}^{-1}$ & $23 / 100 \times 10^{30} \mathrm{~cm}^{-2} \mathrm{~s}^{-1}$ & $\times 1.4 / 3.7$ \\
IP beta function $\beta_{\mathrm{X}}$ & $1.75 \mathrm{~m}$ & $1.25 \mathrm{~m}$ & \\
IP beta function $\beta_{\mathrm{y}}$ & $7.0 \mathrm{~cm}$ & $4.0 \mathrm{~cm}$ & \\
\hline
\end{tabular}

\subsection{LEP: The negative lessons}

LEP's tunnel, the longest ever built, prior to the channel tunnel that links France and England, was carved by three tunnel-boring machines. Underneath the Jura Mountain, the rock had to be blasted because it was not suitable for the tunnelling machines. Disaster struck just two kilometres into the three-kilometre stretch of tunnel in the foothills of the Jura. High-pressure water gushed into the tunnel and formed an underground river which delayed the completion of the tunnel by six months. The lesson here is to avoid tunnelling in regions which are prone to having underground rivers or lakes. This was one of the boundary conditions imposed on the geological survey [1] initiated for the European Study Group preparations in 2013.

A second lesson concerns the value of gathering expertise and carefully scheduling the future runs of these machines. The initial operation of LEP was significantly slowed down by the absence of robust and reliable application software. This lack was rectified during the first winter shut down by a small team of operations experts [2].

Initially, the beam diagnostics for LEP was not fully developed and integrated into the control system. Again, this deficiency was gradually corrected after the initial commissioning [3].

Early in the commissioning, it was observed that performance was limited by strong betatron coupling caused by a thin magnetised nickel layer on the vacuum chamber. This layer was introduced during manufacture in order to bind the lead shield to the aluminium chamber. Fortunately, an in situ demagnetisation technique was developed and successfully deployed [4].

Another challenge was the gross underestimation of the lead time needed for the design, development, construction and reliable operation of the LEP 2 superconducting cavity system.

The impact of the large amount of synchrotron radiation on the beam surroundings was also under-estimated, in particular, the impact on vacuum joints, etc.

\subsection{LEP operational performance}

LEP operated in different modes, with different optics, at different energies, and delivered excellent performance that enabled a rich research programme and contributed to the establishment of the standard model. LEP surpassed all design parameters and expectations (see Table 2), in particular, the peak luminosity at LEP2 was almost a factor of 4 above design. 


\section{The SSC and the LHC}

The superconducting super collider (SSC), with a circumference three times larger than the existing CERN tunnel (LEP) to house the large hadron collider was proposed around the same time. Hence, the collision energy of the SSC was much higher than anything which could be reached by the LHC. The only way in which the LHC could be competitive was by proposing a much higher luminosity. The design luminosity for the LHC was purported to be 10 times higher than that of the SSC, a risky decision since it was not proven at the time that such a high luminosity was achievable. This was a courageous and visionary decision which paid huge dividends to CERN and the world of particle physics by allowing the discovery of the Higgs boson. Another audacious but necessary design option was the choice of the high value of the peak magnetic field in the LHC design which was significantly higher than that of the SSC.

In November 1988, it was announced that a site at Waxahachie, Texas, a town about 25 miles southwest of Dallas, had been ranked outstanding on every major criterion by the site selection committee. In addition, the state of Texas had promised to contribute one billion dollars to the project.

This was a very interesting period for colliders: LEP was already up and running and from a luminosity point of view was outperforming the Californian Stanford Linear Collider (SLC). However, it should be said that the SLC later had greatly improved performance and operated with polarized beams. On the other hand, SSC had received US governmental approval which would surely preclude any form of approval for the LHC, since the world's physicists only needed one super collider. This did not look very encouraging for the future of the world's leading high-energy physics laboratory, CERN.

Following the announcement of the site selection, a second 'site specific' review of the SSC was organised for July 1990. In September 1990, the conclusions made by the review committee were reported, and the shocking outcome was that the total project cost had risen to $8.3 \mathrm{~b} \$$ from the original conceptual design report value of $3.1 \mathrm{~b} \$$ in 1986 . Following further cost escalations in 1991 and 1992, the US congress cancelled the SSC project in 1993 prompting the approval of the LHC in 1994 (see Table 3). The immediate lesson to be learned is obvious, but one may wonder which/what were the mistakes made with the SSC?

Clearly, the cost escalations were the main reasons for the cancellation of the project. In my opinion, the reason behind the cost escalation was that the project had been approved before the design had been completed. This led to costly design changes following the premature approval of the initial design. In addition, it was clear that the SSC did not have the unanimous support of the US HEP community. There was a strong rival group proposing a high-energy linear collider (NLC). Finally, there was the decision about the site location in Waxahachie, Texas, which came as a huge surprise, as the Fermilab site was considered by many to be the preferred one and the optimum choice to serve the scientific community that would use the new facility.

\subsection{SSC site selection lesson}

I firmly believe that if the Fermilab site had been chosen for the SSC, the project would have been a big success for US particle physics. The Waxahachie site had many disadvantages. An unattractive location for families offering limiting opportunities for families and individuals and certainly lacking the atmosphere of an international environment to welcome researchers from around the globe. This further complicated the recruitment of the world's top accelerator engineers and high-energy experimental physicists. 
Table 3 Chronology of colliders

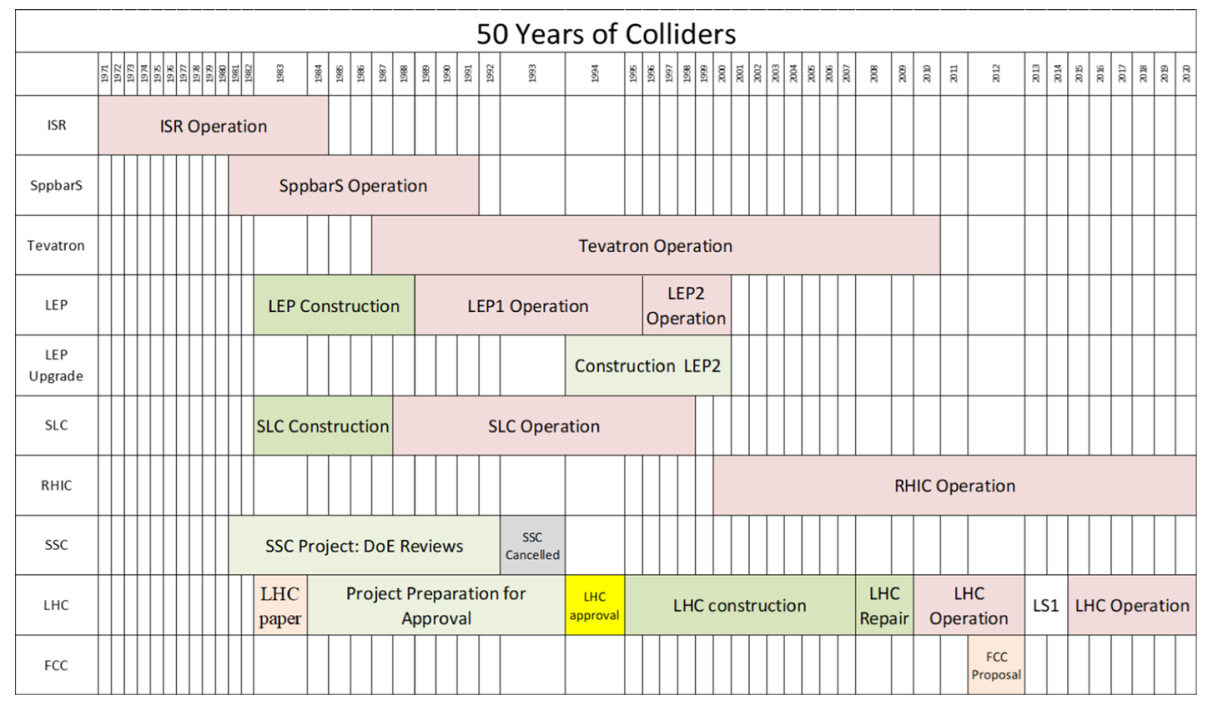

Possibly, more importantly, Waxahachie was a 'green field' site without existing accelerators and poor infrastructure. The development of an accelerator complex like that of Fermilab, Stanford, Brookhaven and CERN takes decades. As an example, see Fig. 1 which indicates the evolution of the CERN accelerator site by giving the construction year for each of the accelerators. As an example, the PS came into operation in 1959 and has been the injector for the CERN Intersecting Storage Rings (ISR), the Super Proton Synchrotron (SPS), the Super proton-anti-proton collider (SPPS), the large electron-positron collider (LEP), and the large hadron collider (LHC) as well as providing beams for its own experimental areas such as neutron time of flight (n-TOF), fixed target experiments in the east area, and the anti-proton facilities.

\subsubsection{LHC}

The LHC is arguably the most successful collider in the history of particle physics due to the discovery of the Higgs boson. The LHC was approved at the end of 1994 following the cancellation of the SSC in 1993. It can be seen from Table 3 that this was an extremely busy time for the CERN technical staff with the initial LHC construction phase competing for human and financial resources with the operation of LEP, the construction project of LEP2 and the operation of LEP2. It is also generally accepted that even when there is an agreed allocation of resources, in the event of faults, the operational project usually attracts priority over the future project due to the urgency of repair(s)/maintenance.

In addition, the LHC had been approved under inadequate funding conditions, with no additional funding for the construction of the largest scientific instrument ever built. The LHC was to be constructed from the annual operations/operational budget of CERN. In fact, in the budget year following LHC's approval, the CERN annual budget was globally reduced by nearly $10 \%$. Add to this the fact that in order to be competitive with the SSC, the LHC performance specifications, in terms of beam energy and peak luminosity, had been pushed to the limit, and possibly beyond. The result of all this was that LHC, an ambitious project 


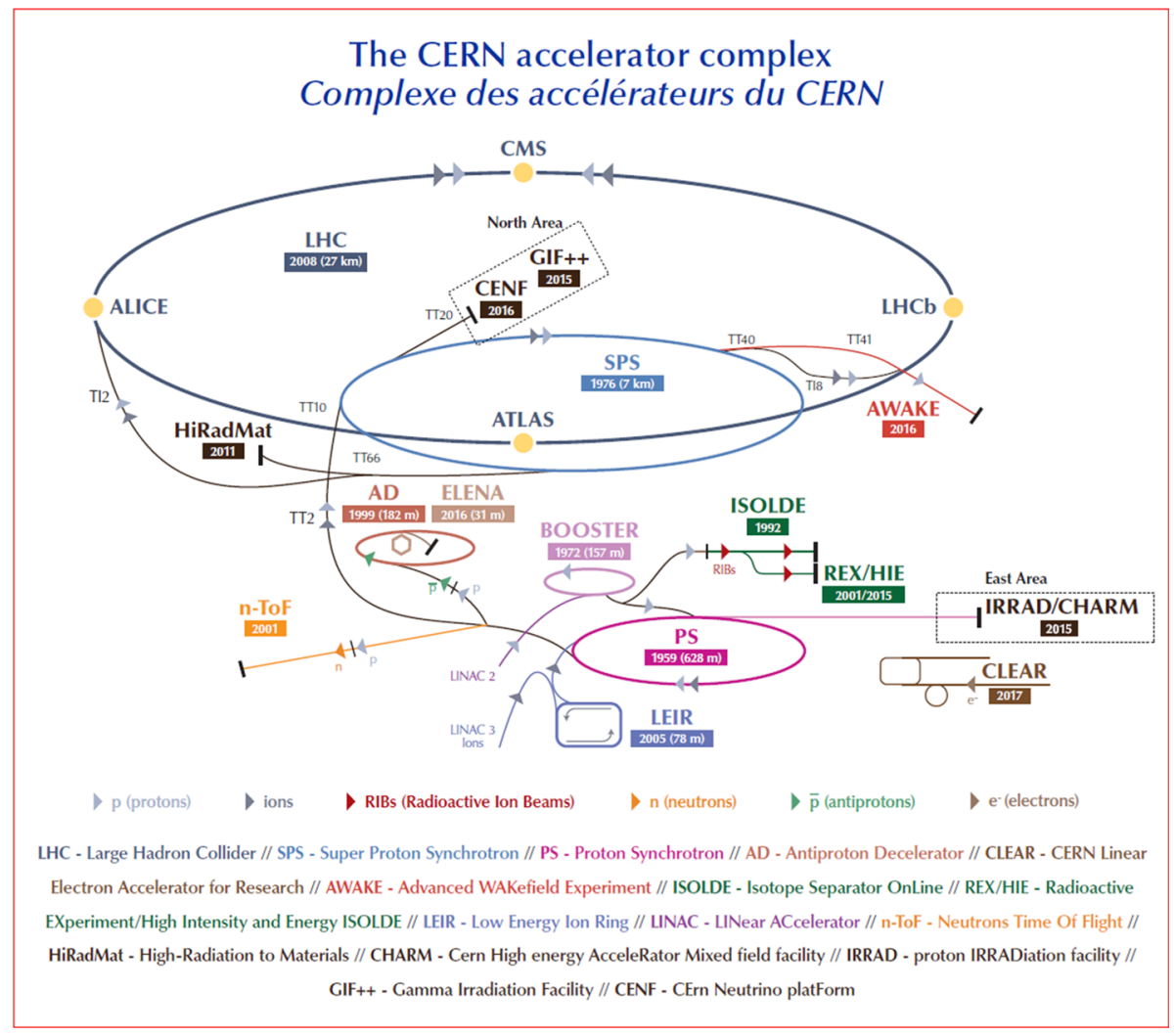

Fig. 1 Schematic of CERN accelerator complex

even with full funding, was grossly under-resourced from 1994 until the closure of LEP2 in 2000. In my opinion, the two conditions mentioned before were the instigators of the many technical crises which LHC encountered: cracks in the welds of the load-bearing structure of the ROCLA magnet handling robots [5], vacuum failures in the cryogenic transfer line (QRL) [6], failure of the support structure in an inner triplet magnet in point 5 [7], and failure of the sliding contacts in the plug-in modules in the interconnects [8]. The lack of funding also meant that resources were concentrated on the technical challenges and less on professional project management including documentation, rigorous testing, and control of sub-contractors.

Following the closure of LEP2, there was massive redeployment of skilled and experienced CERN staff to the LHC project. They joined the LHC well-equipped with their years of experience gained in operating a very large collider which was invaluable for the preparation of the LHC. Their experience had taught them the importance of cold checkout, preventative maintenance, remote repairs and shutdown planning, which are all important aspects of any future collider. The experience gained in operating large superconducting and cryogenic systems also proved invaluable for future LHC operations. 


\section{Roadmap for success}

The boundary conditions for approval of very large and costly projects have evolved since the approval of the LHC. The global media attention received by the LHC has also gained CERN many admirers/supporters throughout the world. The brand name of CERN is of huge importance for any future large-scale project. Nevertheless, in the present social and political climate, we must be aware of and be prepared for, the negative reactions of the media and the general public, which can arise following the proposal of a new multi-billion project. These reactions will not be based on the technical merits or scientific value of the project but on societal, political, and financial considerations.

A recent independent cost benefit analysis of the HL-LHC/FCC [9] has demonstrated the great socio-economic benefit of these projects. The studies revealed six economically relevant benefits:

- the value of scientific publications,

- technological spill over,

- training and education,

- cultural effects,

- services for industries and consumers and

- the value of knowledge for society.

\subsection{Societal}

The purpose of the FCC-ee is to study in greater depth the properties of recently discovered particles like the Z, W, Higgs and the top quark. It is very difficult to convince the non-physicist 'man-in-the-street' that this knowledge justifies the spending of billions of \$US or $€$. We must be aware of the ignorance or lack of interest in high-energy physics and promote the additional, alternative justifications such as spin-off, technology transfer to industry, as well as incentives to young students to study science, technology, engineering and mathematics (STEM) subjects at school and at university. The pursuit of these STEM subjects is crucial for the technical and financial development of the world as we know it today.

There is a huge upsurge, particularly by the younger generation(s), in awareness of climate control/change and its possible disastrous effects in the not-too-distant future. This reality is recognised in the recent update of the European Strategy calling the HEP community to contribute to reducing or offsetting its environmental impact when discussing and planning future facilities. It falls as our responsibility to develop technologies that will reduce the project energy consumption of the FCC while also ensuring that the required electrical energy/power comes from environmentally friendly power sources. It is important to underline that investments in dedicated R\&D for energy efficiency techniques will already pay off in the medium term, with significant impacts on the operating costs of the planned accelerators.

The brand name of CERN in terms of scientific success, technical prowess, and openness are essential ingredients for the success of the FCC proposal. CERN has highlighted the engineering expertise needed for the manufacture of the components needed for these largescale high-tech colliders. This has increased the international competitiveness of the stateof-the art companies which bid for these components.

\subsection{Colliders for peace}

The initial mission of CERN was to unite European countries by collaboration in physics following WW II. The new mission is to unite countries of the World in new high-tech 
physics projects. The SESAME project [10] is a recent example of the power of scientific collaboration among scientists of different religions and nationalities.

In recent years, we have witnessed global turmoil, first in the form of the 2008 financial crisis and more recently in the health pandemic. Both of these dramatic events required global coordination of laboratories, research and governments, thus reminding us of the value of the CERN example and big science in the twenty-first century. CERN's origins can be traced back to the aftermath of the Second World War, when a small group of visionary scientists and public administrators, on both sides of the Atlantic, identified fundamental research as a potential vehicle to rebuild the continent and to foster peace in a troubled region since its birth CERN holds on to its dual mandate to provide excellent science and to bring nations together.

Due to its size and cost, the FCC could be the first intercontinental collaboration project in high-energy physics. The CERN constitution is an excellent example of the kind of collaboration agreement to be sought for this world-wide project. CERN has also been the role model for other big science organisations with which it has strongly collaborated. These include ESO, ESA, SKA, ESS and ITER to name but a few. The value of carefully drafted collaboration agreements is evidenced by many of these projects. It is also clear that wherever possible a central financial budget, like that of CERN, is to be preferred to a high proportion of the budget by 'in-kind' contributions.

\subsection{Technical}

The FCC-ee proposal is technologically ambitious but with greatly reduced risk due to the vast experience gained by other colliders like LEP2. The fundamental limitations and challenges of the FCC-ee, as part of an integrated programme leading to FCC-hh, are discussed in great depth in the essays collected in this volume. The FCC project is also sufficiently flexible to be capable of embracing and profiting from new ideas and technologies which may not yet have been invented.

The use of the existing infrastructure and well-tested pre-accelerators on the CERN site is a huge financial and technical advantage.

FCC-ee will produce an abundance of high-power synchrotron light. It is appropriate to investigate if this synchrotron radiation is interesting in itself, or if the properties of the synchrotron light could be modified in certain areas of the FCC so as to be useful for other applications or science.

\subsection{Financial and project management}

The FCC is a huge financial project and will require specialised financial and commercial project management. For the LHC, earned value management was only introduced several years after the project had been approved. Financial and project management are now essential units in all projects and particularly in high-tech big science projects. FCC will require the setting up of these activities in the very early stages of the project.

In my view, the project management office (PMO) is the backbone of a successful management approach in any organisation and requires specialised tools to provide a list of critical services to the project to:

- Ensure that the right decisions are made by the right people based on the right information. This may include auditing and peer reviews, development of the project structure, validating it and ensuring accountability.

- Provide information that is relevant and accurate to support effective decision-making. 
- Maintain a repository of learned lessons, offering templates and best practices from previous successful projects.

- Facilitate project teams and help them do their jobs more effectively by streamlining process and bureaucracy, offering training, mentoring and quality assurance.

- Manage documentation, project history and organisational knowledge.

It is also important that the FCC profits from practice/applications in other fields. One comparable field is the construction of medical devices such as MRI, PET scanners, CT scanners, radiography devices and proton therapy devices. The regulatory practice in this domain goes a long way towards avoiding errors in device production. Another important aspect is the cost reduction coming from the large-scale production. As an example, it is common that the cost of production of a proton therapy system is reduced by around $60 \%$ from the first to the sixth system. Clearly, we are not going to large-scale produce FCCs! However, there are sub-components in the FCC which are numerous and identical (e.g. short straight sections) which can profit from these 'batch' production techniques and reduce the project cost.

\section{Summary}

In this essay I have looked back, based on my own personal experience, at several aspects of some previous colliders and tried to extract the positive as well as the negative lessons learned in terms of their design, construction, installation, commissioning and operation.

These lessons included the following aspects:

- choice of location.

- control and availability of resources (human \& financial).

- professional 'commercial' project management.

- building an international collaboration with stakeholders from academia and industry.

- the wider socio-economic benefits of a new research infrastructure and the benefits that stem for society as well as the industrial and cultural value of such big science projects.

I have also commented on the relative technological 'leaps' made by each new project in terms of beam energy and machine circumference over the past 50 years or so.

Based on the lessons learned, I have suggested a 'Roadmap for Success' that may help to avoid the pitfalls of previous projects.

Funding Open access funding provided by CERN (European Organization for Nuclear Research). This project is co-funded from the European Union's Horizon 2020 research and innovation programme under grant agreement No 95175 .

Data Availability Statement This manuscript has associated data in a data repository. [Authors' comment: All data included in this manuscript are available upon request by contacting the corresponding author.]

Open Access This article is licensed under a Creative Commons Attribution 4.0 International License, which permits use, sharing, adaptation, distribution and reproduction in any medium or format, as long as you give appropriate credit to the original author(s) and the source, provide a link to the Creative Commons licence, and indicate if changes were made. The images or other third party material in this article are included in the article's Creative Commons licence, unless indicated otherwise in a credit line to the material. If material is not included in the article's Creative Commons licence and your intended use is not permitted by statutory regulation or exceeds the permitted use, you will need to obtain permission directly from the copyright holder. To view a copy of this licence, visit http://creativecommons.org/licenses/by/4.0/. 


\section{References}

1. C. Waaijer et al., Civil engineering feasibility studies for future ring colliders at CERN. in Proceedings of IPAC 13, Shanghai, China, pp 969-971, paper MOPWO036, http://jacow.org/IPAC2013/papers/ MOPWO036.pdf

2. R. Bailey, A. Belk, P. Collier, M Lamont, G de Rijk and M. Tarrant, The impact of new accelerator Control Software on LEP Performance. in Proceedings of PAC'93, Washington D.C., USA, May 1993, pp 1937-1939, http://jacow.org/p93/PDF/PAC1993_1937.PDF

3. M. Lamont, Incorporation of BI equipment into SloppySoft, in Proceedings of the Third Workshop on LEP Performance, Chamonix, January 1993, pp 209- 214. http://cds.cern.ch/record/248984/files/cern-sl93-19.pdf.pdf

4. J. Billan, J.-P. Gourber, J.-P. Koutchouk and V. Remondino. Suppression of the main LEP coupling source. in Proceedings of PAC'93, Washington D.C., USA, May 1993, pp. 68-70. http://jacow.org/p93/ PDF/PAC1993_0068.PDF

5. "ROCLA robots repaired after tough times", CERN Bulletin, 19 July, 2004, http://cds.cern.ch/journal/ CERNBulletin/2004/29/News Articles/768810?ln=en

6. K. Brodzinski et al., Failure mechanism and consolidation of the compensation bellows of the LHC cryogenic distribution line. Phys. Proced. 67, 129-134 (2015). https://doi.org/10.1016/j.phpro.2015.06. 023

7. "Fermilab Update on Inner Triplets", Fermilab Today, April 27, 2007. https://www.fnal.gov/pub/today/ Magnetupdate2.html

8. K. Artoos, M. Guinchard and T. Renaglia, "Sliding force measurements on the LHC contact RF contact plug in modules at $15 \mathrm{~K}$ and in UHV", in Proceedings of EPAC'08, Genoa, June 2008, http://jacow.org/ e08/papers/TUPD021.pdf

9. A. Bastianin and M. Florio, Study of the socio-economic impact of CERN HL-LHC and FCC. in CERN-ACC-2018-0014, CERN, Geneva, May 2018, http://cds.cern.ch/record/2319300/ files/CERNACC-2018-0014.pdf

10. The SESAME Project, https://www.sesame.org.jo/about-us/what-is-sesame 